\title{
THE NATURE OF THE ULTIMATE MAGNETIC PARTICLE.
}

By K. T. Compton and E. A. Trousdale.

Introduction.-For many years scientists have agreed in ascribing the magnetic properties of bodies to the action of exceedingly small elementary magnets, but the nature of these ultimate magnetic particles has remained an open question. This theory in its earliest form was due to Weber, who supposed that the molecules of substances are magnetic doublets whose axes are arranged at random in the unmagnetized substance but, in opposition to restoring forces of elastic nature, are so directed by an external field that the substance acquires a definite resultant magnetic moment. Wiedemann suggested that the opposing couples are of a frictional nature, thus accounting for hysteresis but failing to explain magnetization in very weak external fields. Ewing then suggested that the only restraints on the molecules are due to the mutual magnetic forces occurring between neighboring molecules and showed that the residual magnetism and hysteresis of ferromagnetic substances may be thus accounted for. None of these theories accounted for diamagnetism. A development of Weber's theory, due to Ampère, is that the magnetic properties of molecules are caused by electric currents circulating about perfectly conducting circuits within the molecules. Maxwell showed that, when placed in an external magnetic field, the induced currents set up in these molecules would tend to oppose the field and thus give to the substance diamagnetic properties, while the change in the orientation of the molecular circuits would give rise to paramagnetic properties. Thus a substance is dia- or para-magnetic according as the former or the latter effect predominates.

With suitable modifications, Ampère's theory of molecular currents has been made the basis of the electron theory of magnetism, whose development is due largely to Langevin and Weiss. On this theory electrons revolving in orbits around the atomic nucleus give rise to magnetic fields and constitute the ultimate magnetic particles. In the presence of an external field the magnetic moments of the electron orbits are diminished in the direction of the external field by an effect analogous to electromagnetic induction, so that the resultant effect is of a diamagnetic nature. If the resultant magnetic moment of the electron 
orbits in each atom be different than zero, the planes of the orbits will be oriented so that the magnetic axis of each atom will tend to point in the direction of the external field. This gives rise to paramagnetism, whose intensity depends on the strength of the external field, the magnitude of the resultant magnetic moments of the atoms, the interatomic forces of electrostatic or other nature and the demagnetizing effect of the kinetic reactions due to thermal agitation. Ferromagnetic substances are those in which the resultant magnetic moments of the atoms are so large that all other interatomic forces are negligible in comparison.

A recent theory ${ }^{1}$ suggests that certain numerical relations are most readily explained on the assumption that the ultimate magnetic particle is a natural unit, called the magneton. At present this must be regarded as a mere hypothesis, with a possible basis of fact, since its usefulness in explaining magnetic phenomena is limited to a very small number of phenomena.

The electron theory has been generally accepted as offering the most reasonable explanation of magnetic phenomena and is strongly supported by its success in accounting for the Zeeman effect. Yet there are several magnetic phenomena which apparently receive the readiest explanation on the molecular theory. The most significant of these are: (a) The influence of temperature, a molecular effect, on the magnetic properties of substances; $(b)$ the effect of a changing magnetic field on the temperature, $i . e$., on the average kinetic energy of the molecules of substances; (c) the effect of chemical combination, as in the case of iron salts and Heusler alloys; $(d)$ the effect of mechanical jarring on the ease with which certain substances may be magnetized.

The recently developed method of determining the positions of atoms within a crystal by X-ray photography and the ferro-magnetic properties of magnetite, hematite and pyrrhotite crystals suggested a direct experimental method of eliminating one or the other of these theories. The magnetic properties of these crystals have been investigated by Weiss, ${ }^{2}$ who found that these crystals may be magnetized strongly in the direction of each crystal axis, but that the degree and ease of magnetization is different along different axes. It is practically certain that the magnetic properties of iron and other substances are determined by the properties of the agglomeration of minute crystals of which they are composed. Thus the magnetic properties of crystals are typical of those of all magnetic substances.

We accordingly passed a beam of X-rays through crystals and obtained

1 Weiss, Le Radium, Vol. 8, p. 30I, rgr I.

${ }^{2}$ Journal de Physique, 3, 5, p. 435, I896; 4, 4, p. 469, 1905. 
on photographic plates the diffraction patterns which were determined by the arrangements of the atoms within the crystals. By comparing the photographs taken through the unmagnetized crystals with those taken through the same crystals when magnetized to various intensities it was possible to determine with certainty whether or not the atoms of a substance experience a translational displacement in the process of magnetization. We have found that no such motion occurs and hence that the molecular theory of magnetism, at least in its ordinary form, is untenable.

Apparatus and Method.-A powerful self-regulating X-ray bulb was enclosed in a lead box $E F$ in such a position that a pencil $a b$ of X-rays passed out through an opening $H$, through pin holes in the lead shields $L L^{\prime}$, through the crystal $C$ to the photographic plate $P$. The crystal was cemented by "Quixo" cement to one of the adjustable pole pieces $S$ of an electromagnet capable of setting up a field of $\mathrm{I}, \mathrm{ooo}$ gauss in the air gap. The other pole piece was prevented from touching the crystal in order to obviate the danger of a mechanical displacement of the crystal when strongly magnetized. The apparatus was enclosed in the light

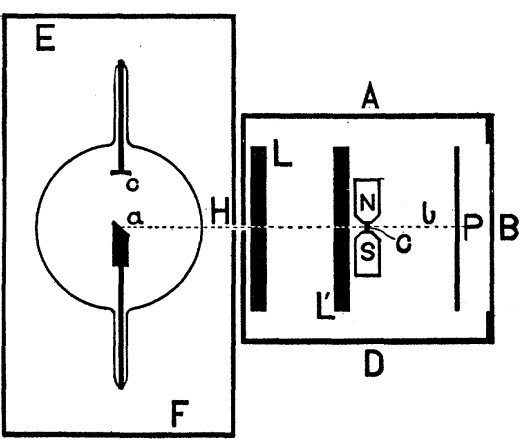

Fig. 1.

tight box $A D$ with a removable back $B$. This box was made of sheet iron in order to reduce the effect of the magnetic field on the path of the cathode rays from $c$ to $a$. Cramer X-ray plates, "New Process," were used, and the negatives were later intensified. The distance of the plate from the crystal was three inches and the exposures were for three hours.

The procedure was to take a photograph through the unmagnetized crystal, then, without disturbing the crystal, turn on the current in the electromagnet and take a second photograph with the crystal magnetized, then take a third photograph with the direction of the magnetic field reversed and finally take another photograph with the crystal unmagnetized. This last photograph was a check to guard against possible effects 
due to any change in the position of the crystal. Supplementary experiments showed us that in every case the magnetic fields were of sufficient strength to strongly magnetize the crystal.

In this way a number of series of photographs were taken through crystals of magnetite and hematite variously oriented with respect to the magnetic field. In no case did we discover that magnetization influenced the diffraction pattern.

Two of these series of photographs, one taken with magnetite $M$ and the other with hematite $H$, are shown in the accompanying figure. (a), (b), (c), (d) refer to the cases of the unmagnetized, magnetized, reversely magnetized and unmagnetized crystals. Unfortunately in bringing out the spots more clearly by intensifying the plates we did not succeed in strengthening the spots equally on the four plates. In the original negatives there was no difference in the cases $(a),(b),(c),(d)$ either in the relative intensities or the positions of the spots. The "fogging" of several of the pictures is also due to the intensifier. We have not as yet succeeded in obtaining specimens of pyrrhotite crystals.

Discussion of Results. - These results show conclusively that the ultimate magnetic particles must be atoms or something within the atoms and are therefore consistent with the electron theory of magnetism. Doubtless a change in the orientation of an atom means the same thing as a change in the orientation of an electron orbit within the atom, since a turning of an electron orbit would upset the equilibrium of the remaining constituents of the atom so that the whole atom would change its orientation. However this may be, it is certain that the ultimate magnetic particle cannot be a molecule or other group of atoms, since the turning of such a system would involve a translational motion of atoms, which the present investigation shows does not exist.

Physical Laboratory,

Reed College. 
Physical Review, Vol. V., Second Series. April, I9I 5.
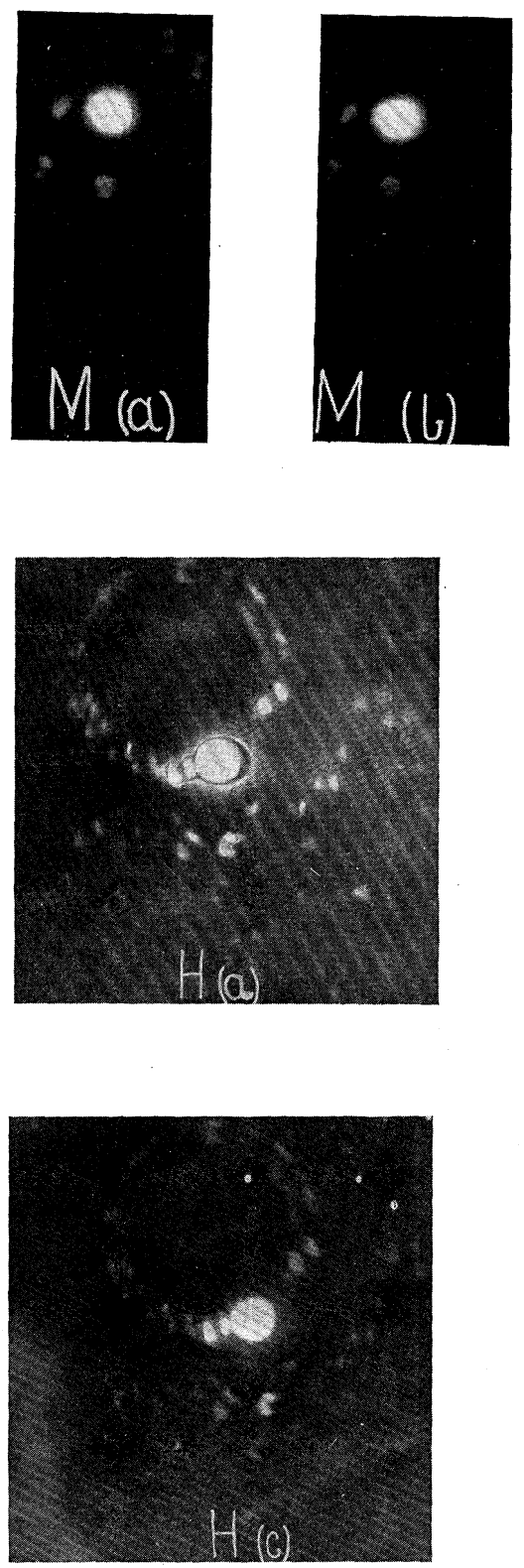

Plate I.

To face page 318 .
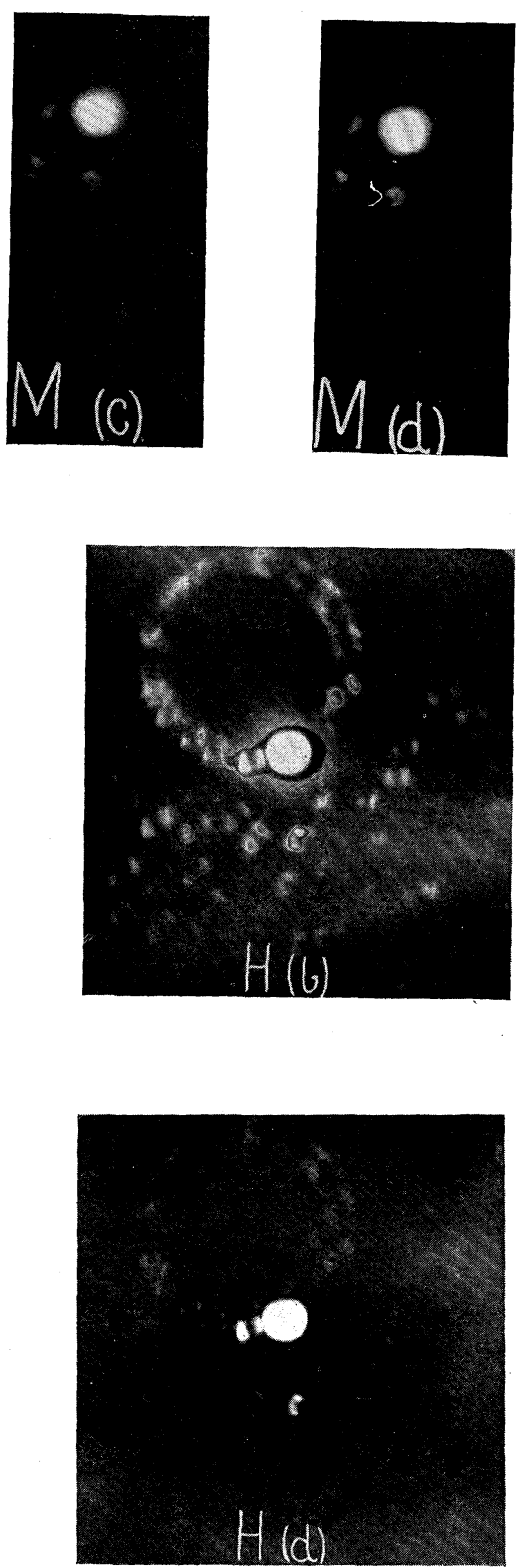

Fig. 2. 

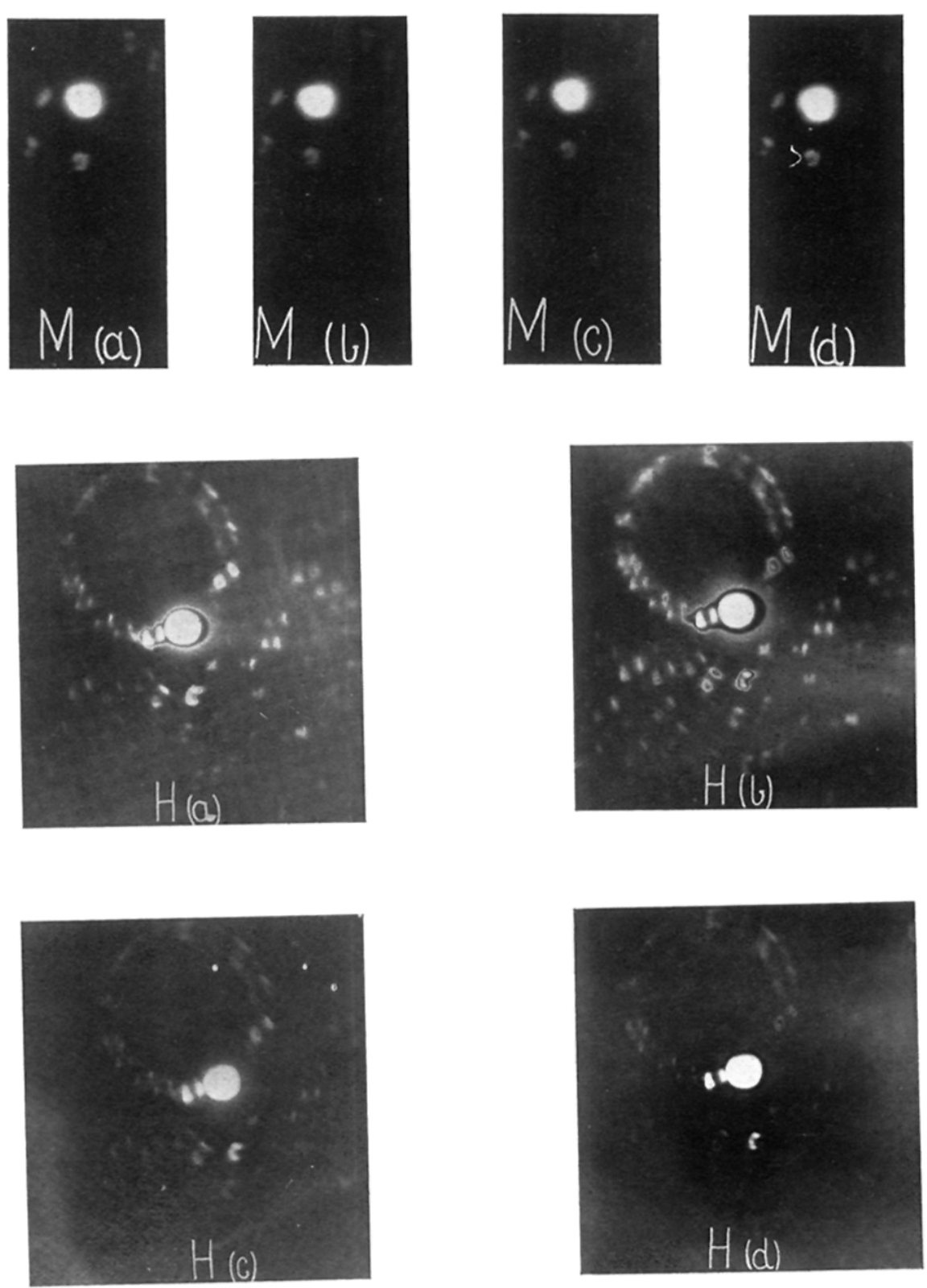

Fig. 2. 